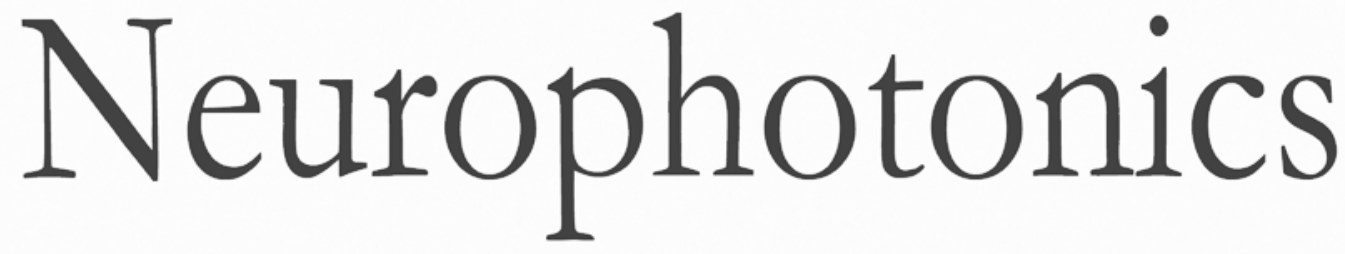

\title{
Optical-imaging-based neurofeedback to enhance therapeutic intervention in adolescents with autism: methodology and initial data
}

Ning Liu

Sarit Cliffer

Anjali H. Pradhan

Amy Lightbody

Scott S. Hall

Allan L. Reiss 


\title{
Optical-imaging-based neurofeedback to enhance therapeutic intervention in adolescents with autism: methodology and initial data
}

\author{
Ning Liu, ${ }^{a, \star}$ Sarit Cliffer, ${ }^{a}$ Anjali H. Pradhan, ${ }^{b}$ Amy Lightbody, ${ }^{a}$ Scott S. Hall, ${ }^{a}$ and Allan L. Reiss ${ }^{a, c}$ \\ aStanford University, Center for Interdisciplinary Brain Sciences Research, Department of Psychiatry and Behavioral Sciences, \\ 401 Quarry Road, Stanford, California 94305-5795, United States \\ bUniversity of California, Department of Molecular and Cell Biology, 142 LSA \#3200, Berkeley, California 94720, United States \\ 'Stanford University, Department of Radiology, 300 Pasteur Drive, Stanford, California 94305-5105, United States
}

\begin{abstract}
Impaired facial processing may contribute to social dysfunction in certain individuals with autism spectrum disorder (ASD). Prior studies show that electroencephalogram-based and functional magnetic resonance imaging-based neurofeedback might help some individuals with ASD learn to modulate regional brain activity and thus reduce symptoms. Here, we report for the first time the feasibility of employing functional near-infrared spectroscopy (fNIRS)-based neurofeedback training in children with ASD. We developed a method to study physiological self-regulation of oxy-hemoglobin using real-time feedback. The paradigm is illustrated with initial data from four subjects who engaged in a facial-identity recognition training program during which an implicit reinforcement was given based on the participant's brain activity and behavioral performance. Two participants had a confirmed diagnosis of ASD, and the other two were typically developing (TD). One participant with ASD and one TD participant received real-feedback (real-FB) during the training, whereas the other two received sham-feedback (sham-FB). After five training sessions, the subjects who received real-FB showed more improvement in facial recognition performance compared with those receiving sham-FB, particularly in the participant with ASD. These results suggest fNIRS-based neurofeedback could enhance therapeutic intervention in children with ASD. () The Authors. Published by SPIE under a Creative Commons Attribution 3.0 Unported License. Distribution or reproduction of this work in whole or in part requires full attribution of the original publication, including its DOI. [DOI: 10.1117/1.NPh.4.1.011003]
\end{abstract}

Keywords: functional near-infrared spectroscopy; neurofeedback; autism; face recognition; implicit reinforcement.

Paper 16024SSR received Apr. 26, 2016; accepted for publication Jun. 13, 2016; published online Aug. 17, 2016.

\section{Introduction}

Autism spectrum disorders (ASDs) are a heterogeneous group of neurodevelopmental disorders including what was previously known as autistic disorder, Rett disorder, childhood disintegrative disorder, pervasive developmental disorder-not otherwise specified, and Asperger disorder. ${ }^{1}$ Individuals with ASD are characterized by deficits in social interaction, communication, and restrictive behaviors (Diagnostic and Statistical Manual of Mental Disorders DSM-5, American Psychiatric Association). These impairments typically arise early in childhood, tend to be chronic, and are typically associated with significant difficulties throughout life for affected individuals. There are various treatment options to address the core symptoms of ASD, including psychopharmacological and behavioral therapies; however, these approaches are sometimes associated with unwanted risks and side effects. In recent years, neurofeedback has gained increasing attention as a noninvasive intervention for children with ASD.

Neurofeedback-based learning studies have been performed using real-time analysis of brain signals with various neuroimaging techniques to enable self-regulation of brain function. Studies show that with real-time feedback of neural signals, subjects can learn to modulate regional brain activity and potentially reduce medical symptoms such as pain, ${ }^{2}$ or improve symptoms of autism. ${ }^{3-7}$ Since the 1960 s, ${ }^{8,9}$ electroencephalogram

*Address all correspondence to: Ning Liu, E-mail: ningl@stanford.edu
(EEG) has been used to provide instantaneous feedback to allow human subjects to develop control of brain activity. In recent decades, this has been extended to several other neuroimaging techniques, such as functional magnetic resonance imaging (fMRI) ${ }^{10-12}$ and functional near-infrared spectroscopy (fNIRS). ${ }^{13,14}$ Functional NIRS-based feedback has several advantages over EEG- and fMRI-based neurofeedback. For instance, it has much better spatial resolution than EEG, and it is easier to locate the signal source from the brain. Functional NIRS uses relatively compact instrumentation, is more tolerant of movement than fMRI, and can be used in a more naturalistic social environment. ${ }^{15}$ Furthermore, fNIRS-based neurofeedback intervention is more cost-effective than using fMRI, especially considering that this type of intervention generally involves several training sessions.

Several studies in recent years have used fNIRS-based neurofeedback as a tool to enable self-regulation of brain function in either healthy or affected populations. ${ }^{13,14,16-21}$ The oxy-hemoglobin (oxy-Hb) signal was predominantly selected as the feedback signal source because of its superior sensitivity in task-related signal changes and its high correlation with blood oxygen level-dependent signals in fMRI. ${ }^{22-24}$ Two basic neurofeedback models were used in these studies. In one model, cortical oxy-Hb signals were processed using a general linear model (GLM), and the calculated $t$-values were used to provide feedback for the subjects. ${ }^{13,16,18}$ In the other model, oxy-Hb signal changes were presented to the subjects directly. The feedback was presented either as a vertical bar with changing 
height and color or as a moving object (fish, dot, or a line plot). The feedback source signals were obtained in several different ways. For instance, some studies detected the feedback source signal at one predefined region of interest (ROI); ${ }^{13,16,18}$ Kober et al. ${ }^{14,17}$ utilized the difference values between two predefined ROIs to reduce the influence of global physiological artifacts; Marx et al. ${ }^{20}$ subtracted the signal from the predefined ROI with the average of all the measured signals. In addition to using a predefined ROI, one study utilized a calibration period before the training task to automatically select an fNIRS channel as the ROI. ${ }^{21}$ Mihara et al. ${ }^{13,16}$ reported that fNIRS-mediated neurofeedback enhanced motor imagery-related premotor activation in both healthy subjects and poststroke participants. In this study, subjects were randomly assigned to real-feedback (real-FB) and sham-feedback (sham-FB) groups to receive six sessions of mental practice with motor imagery. In the realFB group, cortical oxy-Hb signals detected by fNIRS were used to provide feedback for the subjects, while in the shamFB group, irrelevant randomized signals were presented. The results showed greater functional gain and significantly greater cortical activation in the premotor area in the real-FB group than in the sham-FB group. Another study conducted by Kober et al. ${ }^{17}$ confirmed the efficacy of fNIRS-based neurofeedback in healthy subjects with a similar motor imagery task. The results showed focused brain activation over the motor areas in the real-FB group and diffused brain activation in the sham-FB group. Lee et al. ${ }^{18}$ investigated the effect of fNIRS neurofeedback on gait. In this study, four healthy participants performed treadmill walking both with and without feedback. The results demonstrated that neurofeedback induced significantly greater activation in the premotor area and supplementary motor area when compared with the nonfeedback condition. Sakatani et al. ${ }^{19}$ evaluated the effectiveness of an fNIRS-based neurofeedback system that modulated activity in the prefrontal cortex (PFC) of healthy subjects. The results suggested that the neurofeedback system could enhance self-control of PFC activity. A recent study by Hasseini et al. ${ }^{21}$ investigated the feasibility of fNIRS neurofeedback to enhance executive function of healthy adults. Two groups trained with a verbal working memory task with either real-FB or sham-FB in four sessions. The results indicated that the real-FB group had significantly improved executive function performance and significantly reduced training-related brain activity in PFC compared with the sham-FB group. Marx et al. ${ }^{20}$ investigated the feasibility of fNIRS neurofeedback as a method for the treatment of attention deficit/hyperactivity disorder (ADHD). Hemodynamic brain activity in the dorsolateral PFC was measured and fed back in 12 training sessions. They compared the fNIRS neurofeedback with EEG-neurofeedback and EMG-feedback, and the results showed a significant reduction in ADHD symptoms in the fNIRS group but not in EEG and EMG groups.

Although fNIRS-based neurofeedback has been investigated in a few clinical populations such as stroke patients and children with ADHD, it has not been applied to children with an ASD. This study was designed to investigate the feasibility of fNIRS-based neurofeedback intervention for improvement in symptoms of ASD. In particular, we developed a paradigm for enhancing treatment response to a computer-based social cognition training intervention in individuals with ASD.

Previous studies show that impaired face processing may contribute to social dysfunction in some individuals with ASD. ${ }^{25,26}$ Improving face recognition abilities could significantly contribute to improvement in quality of life for these individuals. Further studies have demonstrated that face recognition skills can be enhanced through direct training. ${ }^{27}$ An existing computer program called "Let's Face It (LFI)",28 has been used for enhancing face recognition skills in children with ASD. Accordingly, studies have shown that this intervention program can produce improvements in face recognition skills in children with ASD. ${ }^{29,30}$ Specifically, the recognition of facial features presented in isolation or as a full face (parts/whole identity subtest) produced reliable improvements after $20 \mathrm{~h}$ of training with $\sim 100$ min of training per week. In this study, we developed an fNIRS-based intervention paradigm to enhance the effectiveness of the LFI intervention. To exemplify the feasibility of the feedback and physiological regulation of the fNIRS signal, four children [two with previously diagnosed ASD and two typically developing (TD)] were treated using the paradigm. Each subject participated in five training sessions designed to self-regulate the fNIRS signal in brain areas involved in the facial recognition network. Specifically, brain activation in the prefrontal and temporal cortices was measured. We predicted that children who received the face training intervention combined with fNIRS real-time feedback would show greater performance gains on a facial recognition task compared to children undergoing face training combined with sham-FB.

\section{Methods}

\subsection{Participants}

The study protocol was approved by the Stanford University Institutional Review Board. All participants under 18 years old gave written informed assent, as did their parents or caregivers, and all participants over 18 years old provided written informed consent prior to participation. All subjects received payment at the end of the study for their participation.

To demonstrate the feasibility of the method, four adolescents were recruited to participate in the preliminary study that consisted of five weeks of training intervention. Two subjects had a confirmed diagnosis of ASD and two subjects were TD healthy adolescents. The diagnoses of ASD were confirmed using the Autism Diagnostic Observation Schedule (ADOSII) Module 3 or $4 .^{31}$ Both children with ASD were "high functioning" with full-scale IQs comparable to children with TD, based on the Wechsler Abbreviated Scale of IntelligenceSecond Edition (WASI-II; Wechsler, 2011), and with a parentreported comprehensive reading level of fourth grade or above. The mental age (chronological age $\times$ full scale IQ/100), cognitive skills, and severity of autistic symptoms of the four participants are listed in Table 1.

\subsection{Experimental Procedure}

In each participant's first visit, several standard neuropsychological and behavioral measures were conducted. The presence of ASD was determined using the ADOS-II, the social responsiveness scale (SRS), and the Social Communication Questionnaire (SCQ). The ADOS-II was administered directly to the participants with ASD by a trained experimenter to ascertain ASD diagnostic status. The $\mathrm{SCQ}^{32}$ and $\mathrm{SRS}^{33}$ were completed by the parents or designated caregivers of all participants, and these scores were also used to confirm the diagnosis and severity of autistic behavior in participants with ASD. In addition to the parent report and behavioral measures, the complete LFI skills 
Table 1 Participants' characteristics.

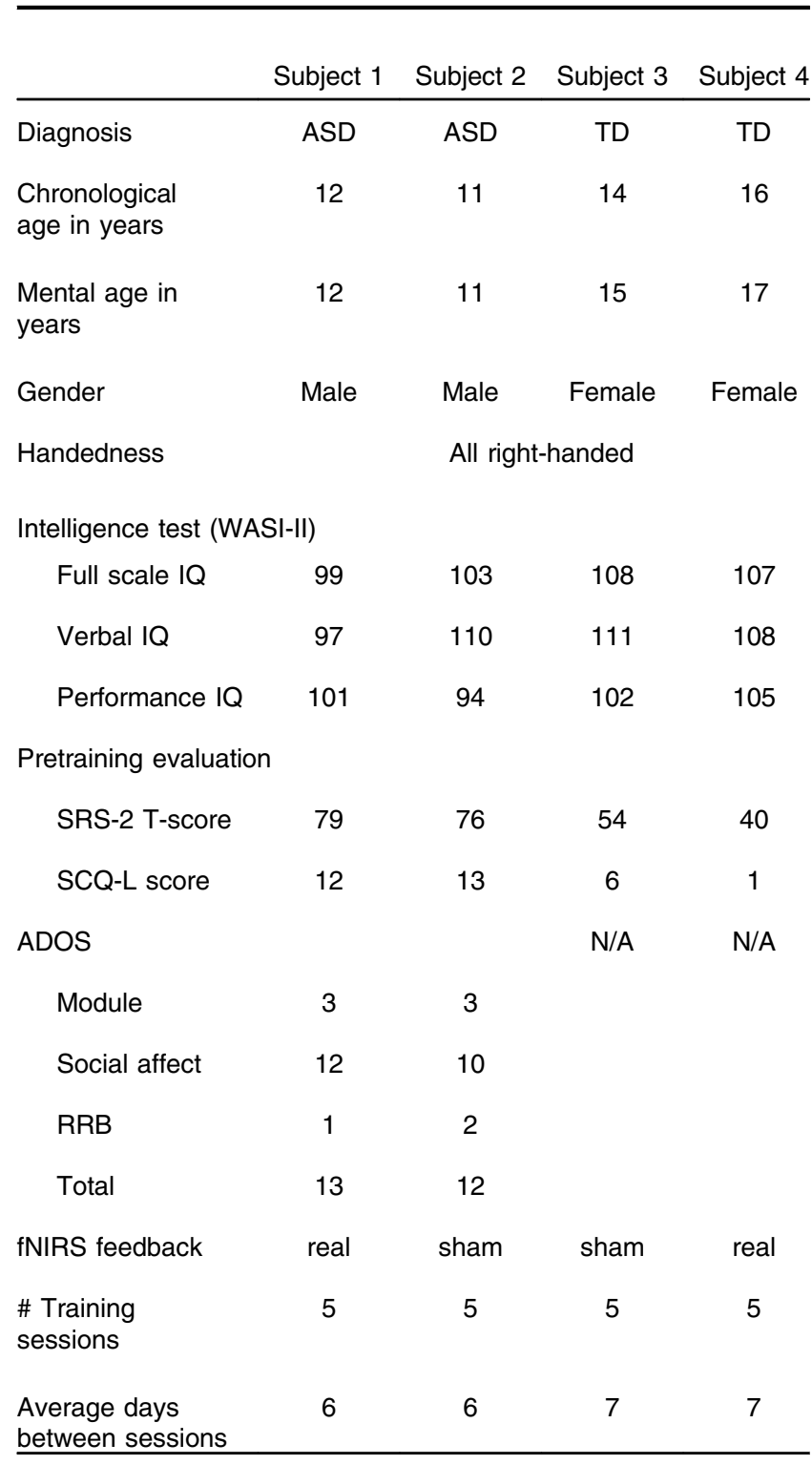

Note: WASI-II, Wechsler abbreviated scale of intelligence-second edition; SRS-2, social responsiveness scale, second edition; SCQ-L, social communication questionnaire, lifetime version; ADOS, autism diagnostic observation schedule; $\mathrm{RRB}$, restricted and repetitive behavior.

battery evaluation ${ }^{30}$ was administered to all participants at their first visits. The LFI skills battery is composed of five tests of facial identity, three tests of facial emotion, and two tests of object processing. In this study, we focused on the parts/whole identity test as one of the criteria for individuals with ASD to participate in the study. Children with an ASD diagnosis were eligible for the study if they scored below $85 \%$ on this test, indicating deficits in facial-identity recognition. Enrolled participants received training for five sessions, with an average of one training session per week. Participants were semirandomly assigned to receive the training with either real-FB or sham-FB (Table 1). After all training sessions, the LFI skills battery evaluation was administered again to all participants to assess their change in behavioral performance.

Instructions were given to the participants at the beginning of each training session by the experimenter. The instruction was based on a PowerPoint file that explained the task with pictures and simple words. Instructions were consistent for all participants whether they received the real-FB or the sham-FB. In particular, the participants were told that bonus points would be determined by the machine automatically based on their brain activity. To increase their engagement in the task, participants were told that the final score of each training session would be converted to real cash as part of their payment.

\subsection{Task Development}

A computer-based task was developed to test and teach the recognition of facial identity utilizing real-time fNIRS neurofeedback. The experiment consisted of a functional localizer task and a facial-identity training task, which ran in sequence. The functional localizer task was used to identify the ROI for each scan. The signal from the determined ROIs was then utilized in the training task to generate the feedback signal in real time. The development of the training task was based on the Face Maker identity subtest of the LFI intervention computer program. ${ }^{29}$ A 5-s instruction screen was shown right after the functional localizer task to indicate the beginning of the training task. The total duration of the task was about 20 min (task design is shown in Fig. 1).

\subsubsection{Functional localizer task}

The functional localization task started with a 20-s rest period. During the rest period, the word "Rest" (in white letters) was displayed at the center of a blue screen. The task was a block design, which consisted of five epochs of emotional dynamic morph faces (MFs) and five epochs of static house pictures (HPs), presented in alternation as follows: MF-HP-MF-HPMF-HP-MF-HP-MF-HP. Each MF epoch contained four dynamic face videos that were randomly selected from a set of eight videos for each run. The videos were generated in FantaMorph (v4.1, Abrosoft $\left.^{34}\right)$ in advance using two male and two female faces. A "happy" and "sad" morph video was created for each face, for a total of eight videos. All images used in these videos were imported from the NimStim set of facial expression stimuli. ${ }^{35}$ Each video lasted for $5 \mathrm{~s}$ and consisted of $3 \mathrm{~s}$ dynamic morph from neutral to full emotional expression (either "happy" or "sad"), followed by $2 \mathrm{~s}$ of the final full emotional expression. The duration of an MF epoch was 20 s. Each HP epoch contained 10 static HPs. These pictures were presented for $2 \mathrm{~s}$ each and contained one house image presented in grayscale on a white background. The HPs were randomized for each training session. The duration of each HP epoch was $20 \mathrm{~s}$. After the last HP epoch, there was a 20 -s rest period. The total duration of the functional localizer task was $240 \mathrm{~s}$. In order to direct the focus of the participants to the screen, a series of red dots with 1-s duration was superposed at the center of the screen. The time interval of the red dots varied between 5 to $25 \mathrm{~s}$, and the participants were instructed to pay close attention to the screen and click the mouse once whenever the red dot appeared.

\subsubsection{Facial-identity training task}

The training task consisted of house-matching (HM), facematching (FM), and bonus display (BD) epochs, presented repeatedly with the following fixed order: HM-FM-BD. Each house epoch lasted about $20 \mathrm{~s}$, each face epoch lasted about $28 \mathrm{~s}$, and each BD epoch lasted about $2 \mathrm{~s}$. There were $30 \mathrm{~s}$ of rest 


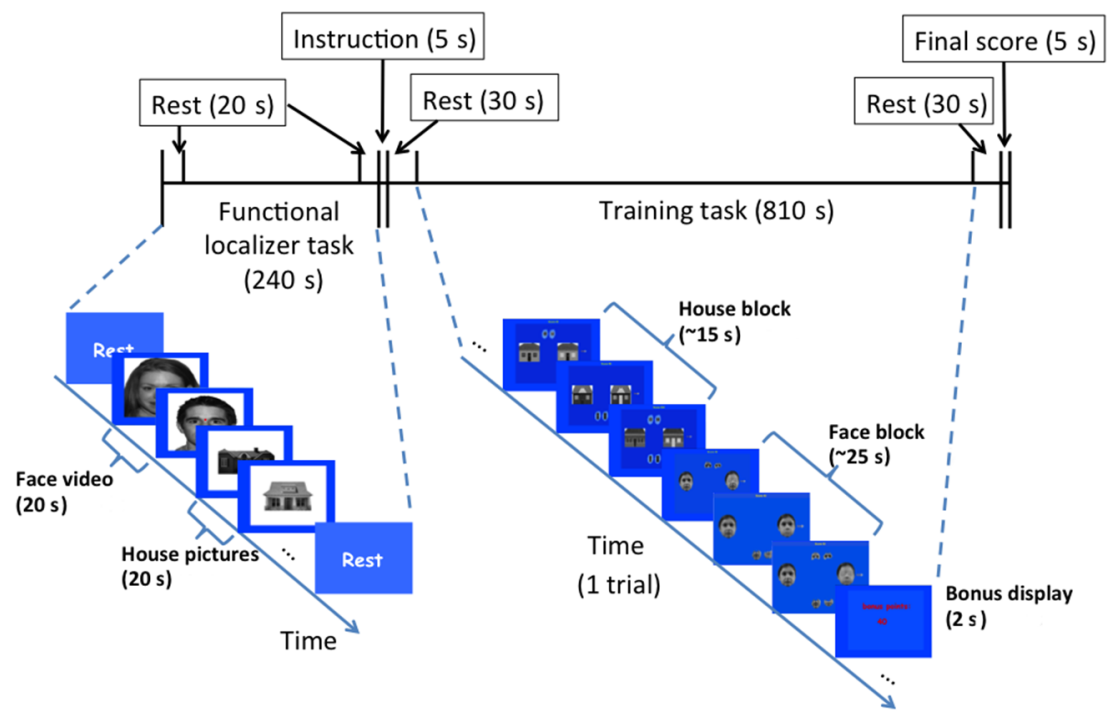

Fig. 1 Task design.

before and after the task epochs. The total duration of the training task was about $810 \mathrm{~s}$. The design of the HM trial was to mimic the FM trials adopted from the LFI intervention computer program.

The HPs used in the training task were generated in a gray color scale. Each house had one door at the center and two identical windows on each side. A trial during an HM epoch started with two houses shown on a blue screen with a complete HP on the left and an incomplete HP on the right. The incomplete house was missing two windows, the door, or all three. A score was presented at the top of the screen, which started from zero and accumulated during the game according to performance. For each missing attribute, the participant was presented with two options to choose from to match the complete house shown on the left. Window options were shown above the houses, and door options were shown underneath; all options were centered between the two houses presented. The participant was instructed to pick the window or the door that matched the complete HP. When the participant clicked on an option, the corresponding missing part would appear on the incomplete house, and they were allowed to switch between options until they were satisfied that the features matched those of the complete house. The participants were instructed to fill in all the missing parts before clicking on the "Done" icon at the very right of the screen, which would lead them to the next trial with either a pair of houses or the same house with different missing parts. If they made a correct choice, the score would increase by 10 points; otherwise, the score would decrease by 2 . An updated score was continuously shown at each trial based on their previous performance.

The face pictures used in the training task were imported from the "LFI" intervention program and were also presented in a gray color scale. In this training program, we were targeting a specific facial recognition impairment associated with autism. These impairments include recognition of identity across changes in facial features, analytic and holistic face processing strategies, and attention to information in the mouth and eye regions. Similar to the HM epochs, a trial in an FM epoch started with two faces shown on a blue screen with a complete face picture on the left and an incomplete face picture on the right, missing both eyes, the mouth, or all three features. The faces were otherwise identical. For each missing attribute, the participant was presented with two options to choose from to match the complete face shown on the left. Eye options were shown above the faces, and mouth options were shown underneath; all options were centered between the two faces presented. The participant was instructed to pick the set of eyes or the mouth that matched the complete face picture. When the participant clicked on an option, the corresponding missing part would appear on the incomplete face. Participants were allowed to switch between options until they were satisfied that the features matched those of the complete face. Just as in the house epochs, the participants were instructed to fill in all the missing parts before clicking on the "Done" icon at the very right of the screen, which would lead them to the next trial with either a pair of faces, or the same face with different missing parts. If they made the correct choice, the score would increase by 10 points; otherwise, the score would decrease by 2 . An updated score was continuously shown at each trial based on their previous performance.

A bonus score was rewarded immediately after each FM epoch and was displayed at the center of a blue screen and flashed between red and yellow for $2 \mathrm{~s}$ to draw the participant's attention to it. The bonus score was then added to the training score, and the total score was displayed in the next task trial. We used the bonus score as the tangible reinforcer to enhance the effectiveness of the participant's neural as well as behavioral performance during the intervention. We designed this incentive feedback instead of continuous real-time feedback to avoid circumstances in which the participant would have to divide his or her attention. This approach, utilizing an embedded (implicit) reinforcer, has the advantage of allowing the participant to continue to attend to the cognitive training task without increasing (explicit) cognitive load.

\subsection{Functional Near-Infrared Spectroscopy Data Acquisition}

Functional near-infrared spectroscopy (fNIRS) signal was acquired using an ETG-4000 Optical Topography system (Hitachi Medico Co., Tokyo, Japan) with a sampling rate of $10 \mathrm{~Hz}$. The measurement patches consisted of an evenly distributed array of 

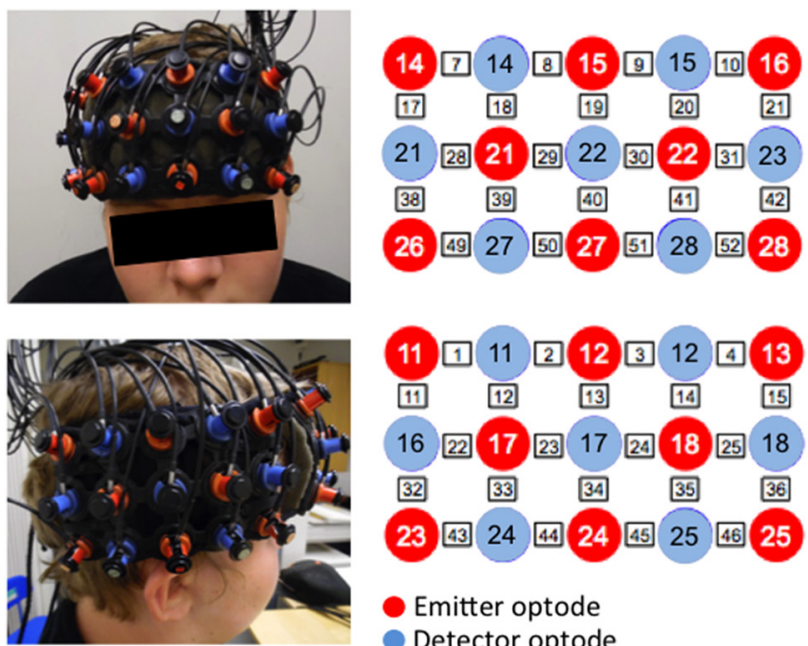

Emitter optode

Detector optode

$\square$ Channel

(a)

(b)

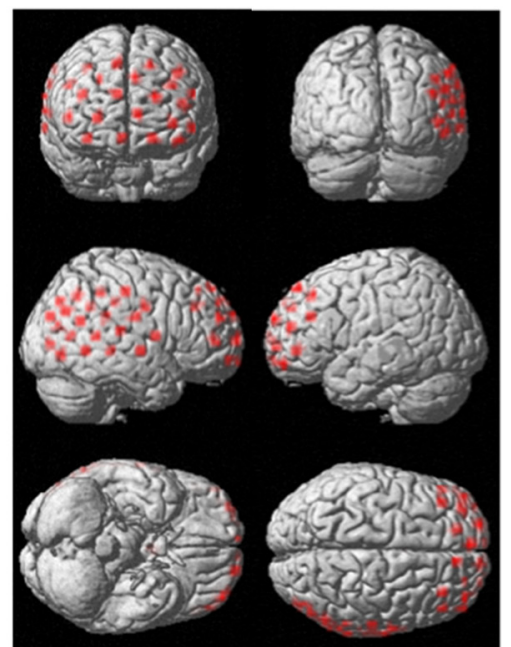

(c)

Fig. 2 Functional NIRS cap placement and the optodes arrangement. (a) The fNIRS cap placement on a representative participant. (b) The optodes and channel arrangement. (c) A representative projection of channel location on a brain template.

alternating emitter and detector fiber bundles (optodes). Optodes were spaced $30 \mathrm{~mm}$ apart, resulting in a spatial resolution of $30 \mathrm{~mm}$ for the system. A channel represented the area measured by one emitter-detector pair, and the channel location was defined as the center position of the emitter-detector optode pairs. A single $3 \times 5$ measurement patch containing 15 optodes was positioned over the PFC of each subject's head, resulting in 22 measurement channels. Another $3 \times 5$ measurement patch was positioned over the right temporal cortex (Fig. 2).

We chose these two regions based on their previously identified roles in cognitive processes involving facial recognition. For individuals with ASD, the face recognition networks usually include aberrant activation in the fusiform face area and significantly increased activation in the PFC, temporal cortex, or primary visual cortex as compared with TD individuals. ${ }^{36,37}$ Among these key brain regions, fNIRS can detect only cortical regions underlying the scalp and skull because of the limited penetration depth of the technique. Therefore, we utilized the brain signal measured from the prefrontal and temporal cortices in our study. The individual locations of the optodes on each participant's head were obtained by a three-dimensional magnetic digitizer system (PATRIOT, Polhemus, Vermont) directly after the task was completed. The technique measured the placement of each optode in relation to five anatomical reference points on the subject's head (nasion, left and right preauricular points, vertex, and inion).

\subsection{Online Functional Near-Infrared Spectroscopy Data Analysis for Region of Interest and Neurofeedback}

The real-time fNIRS signal processing using in-house MATLAB ${ }^{\circledR}$ software primarily included two functions. The first was to determine the ROI from the functional localizer task and the second was to generate the feedback signal during the training task.

Recorded changes in optical density during the localizer (morph) task were first converted into concentration changes of oxy-Hb $([\mathrm{HbO}])$ and deoxy-hemoglobin using the modified
Beer-Lambert law. ${ }^{38}$ The channels that contained extremely noisy signals were determined during calibration before the scan began and were excluded from the later analysis. All [HbO] signals were filtered by a bandpass of $0.01-0.5 \mathrm{~Hz}$ after the morph task to remove high-frequency instrument noise, physiological heartbeat noise, and low-frequency drift before they were input to a GLM. The GLM analysis was performed online for all [HbO] signals. The standard hemodynamic response function (HRF) and two regressors corresponding to the MF condition and the HP condition, respectively, were used in the analysis. The resulting $t$ values of beta weights for the MF condition were used to determine two primary locations for the fNIRS neurofeedback process: (1) the signal corresponding to the highest $t$ value was selected and the channel location of that signal was considered part of the face-processing network at the temporal cortex, denoted by $\mathrm{ROI}^{F}$; (2) the signal corresponding to the lowest $t$ value was also selected and the channel location of that signal was considered irrelevant to the face-processing network and was used as a reference region, denoted by $\mathrm{ROI}^{R}$.

During the training, the bonus score was calculated in real time directly after the completion of each FM epoch for the real-FB participants. To increase the specificity of the feedback and add an internal control, we fed back the differential fNIRS signals from the two regions of interest $\left(\mathrm{ROI}^{F}\right.$ and $\left.\mathrm{ROI}^{R}\right)$ to the participants. This has the advantage of canceling out any global unspecific effects due to factors such as generally increased blood flow. ${ }^{39-41}$ Specifically, we first calculated the percent signal change (PSC) at both ROIs. For instance, the value of the $\mathrm{PSC}$ at $\mathrm{ROI}^{F}$ was defined as the difference between the mean value of [HbO] of an FM epoch and the mean value of [HbO] of the paired HM epoch shown right before the FM epoch, divided by the mean value of $[\mathrm{HbO}]$ of the $\mathrm{HM}$ epoch, i.e., $\mathrm{PSC}_{i}^{F}=$ $\left(\overline{\left[\mathrm{HbO}_{\mathrm{FM}}\right]_{i}}-\overline{\left[\mathrm{HbO}_{\mathrm{HM}}\right]_{i}}\right) / \overline{\left[\mathrm{HbO}_{\mathrm{HM}}\right]_{i}}$, where $i=1,2,3, \ldots, n$ is the index of the FM epochs (which is the same as the index of the HM epochs). Considering the time lag of the hemodynamic response, we removed the first $5 \mathrm{~s}$ of the signal while calculating the mean value for each epoch. Similarly, we calculated the value of PSC $\left(\mathrm{PSC}_{i}^{R}\right)$ at $\mathrm{ROI}^{R}$. Second, we calculated the difference 
between the two PSCs, denoted as diffPSC ${ }_{i}^{\mathrm{FR}}$. When both $\mathrm{PSC}_{i}^{F}$ and $\operatorname{diffPSC}_{i}^{\mathrm{FR}}$ were larger than zero, 40 bonus points were awarded to the participant; when both $\operatorname{PSC}_{i}^{F}$ and $\operatorname{diffPSC}_{i}^{\mathrm{FR}}$ were less than zero, zero bonus points were given; for all other conditions, the participant received 20 bonus points. For the sham group, the bonus points were generated for each training session before the task began: a set of numerical data with six zeros, five twenties, and five forties was randomly permutated before each training session to generate a data sequence to be used for the bonus points. At the end of the training task, the participant's final score was shown on the screen.

\subsection{Outcome Measure}

The effect of fNIRS neurofeedback training on facial-identity recognition was measured using the LFI skills battery evaluation tests. The evaluation was administered pre- and post-training sessions. The number of correct responses per minute (CPM) was used as the primary outcome measure to test participants' improvement in facial recognition performance. The CPM measure was defined as the test score divided by the total reaction time: $\mathrm{CPM}=$ score $/($ sum of reaction time in milliseconds $) \times 1000 \times$ 60. For comparison, we defined the change in CPM as the difference in CPM pre- and post-training sessions, i.e., $\triangle \mathrm{CPM}=$ $\mathrm{CPM}_{\text {post }}-\mathrm{CPM}_{\text {pre }}$.

\subsection{Offline Data Processing and Analysis}

The behavioral learning effect was studied by using the percentage error rate, which was defined as the number of incorrect responses divided by the total number of responses for each training session.

Previous studies show that the posterior superior temporal sulcus (pSTS) is important for face processing. ${ }^{42-44}$ For offline analysis of the fNIRS signal, we focused on changes in [HbO] at the pSTS region during the functional localizer task over five training sessions. Specifically, we first generated a mask image of the pSTS in xjview. To determine the accurate location of the pSTS, we utilized the atlas of the posterior portions of the middle and superior temporal cortices and selected a cluster of voxels that were located at the contacting edges of the two regions. All the voxels that had their $y$-axis below -50 or above -20 in MNI space were removed from the cluster. The remaining voxels were defined as the ROI in the offline analysis and are shown in Fig. 6(c). Considering that the spatial resolution of our fNIRS system was $3 \mathrm{~cm}$, we included all fNIRS channels located within $15 \mathrm{~mm}$ of the defined ROI in the following analysis. Specifically, the $[\mathrm{HbO}]$ signals from those channels were first filtered by a bandpass of 0.01 to $0.5 \mathrm{~Hz}$. The filtered signals that still had a standard deviation of 0.25 or above were considered extremely noisy based on empirical evidence and were removed from analysis. The remaining signals were averaged and input to the GLM. The contrast values were then calculated between MF and HP conditions for all training sessions and compared among participants.

\section{Results}

\subsection{Behavioral Performance}

The changes in the number of correct responses per minute $(\triangle \mathrm{CPM})$ for all four participants are presented in Fig. 3. After five training sessions, all participants were able to improve their performance on the evaluation task. Furthermore, participants

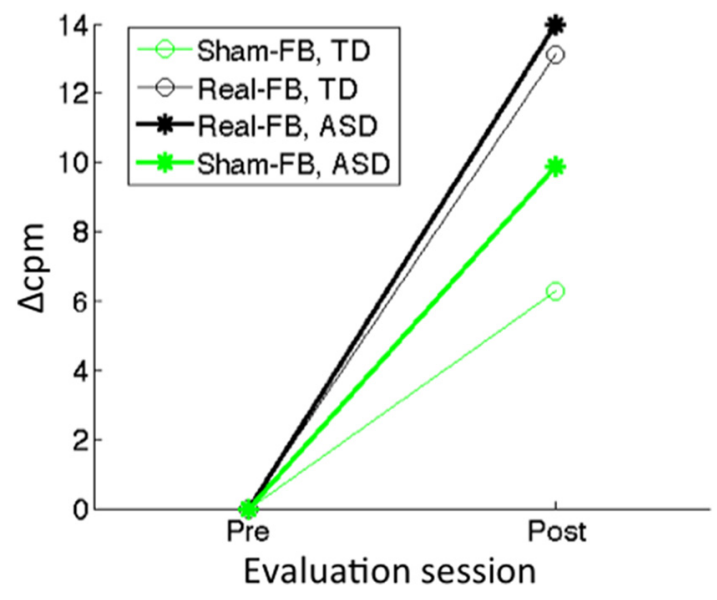

Fig. 3 Changes in the number of correct responses per minute $(\Delta \mathrm{CPM})$ for the four participants.

with real-FB showed increased improvement in CPM as compared to the participants who received sham-FB.

\subsection{Online Data Analysis}

Figure 4 shows the $\mathrm{ROI}^{F}$ and $\mathrm{ROI}^{R}$ for the participants with ASD who received real-FB. The red dots (with black circles) represent the corresponding fNIRS $\mathrm{ROI}^{F}$ channel locations and the green dots represent the corresponding fNIRS $\mathrm{ROI}^{R}$ channel locations. The locations of the red dots were scattered, in particular, the red dot of session 3 was located at the PFC.

\subsection{Offline Data Analysis}

Figure 5 shows the percentage error rate for all four participants. The error rate of FM epochs for the participant with ASD who received real-FB [shown as the magenta line in Fig. 5(a)] was decreased by $25 \%$ across the five training sessions, while the error rate was decreased by $5 \%$ for the participant with ASD who received sham-FB [shown as the magenta line in Fig. 5(b)]. At the fifth training, both TD participants were able to reach an error rate of $2 \%$ or below, while both participants with ASD had an error rate of $5 \%$ or above. The error rates of HM epochs were comparable for all participants (shown as the black lines in Fig. 5).

Figure 6 shows a contrast map of a representative participant and its corresponding fNIRS channel location on the brain template. The map in Fig. 6(b) represents the contrast between the dynamic MF and the static HP conditions during a localizer scan. The blue line in Fig. 6(b) roughly marks the location of the pSTS region that corresponds to the location of the blue line in Fig. 6(a). Notably, the locations of the fNIRS channels were different for each training session, because it was difficult to place the fNIRS optodes in the exact same location for each scan. Thus, the selected channels from ROI region were not the same for each scan session.

The results from the ROI analysis are shown in Fig. 7. The trend lines of the contrasts across the five training sessions for the four participants are also shown. Figure 7(a) shows the change of contrast at the ROI for the two participants with ASD, and Fig. 7(b) shows the change of contrast at the ROI for the two TD participants. The slopes of the trend lines for the two TD participants are both negative and are relatively small compared to the slopes of participants with ASD. The slope of 


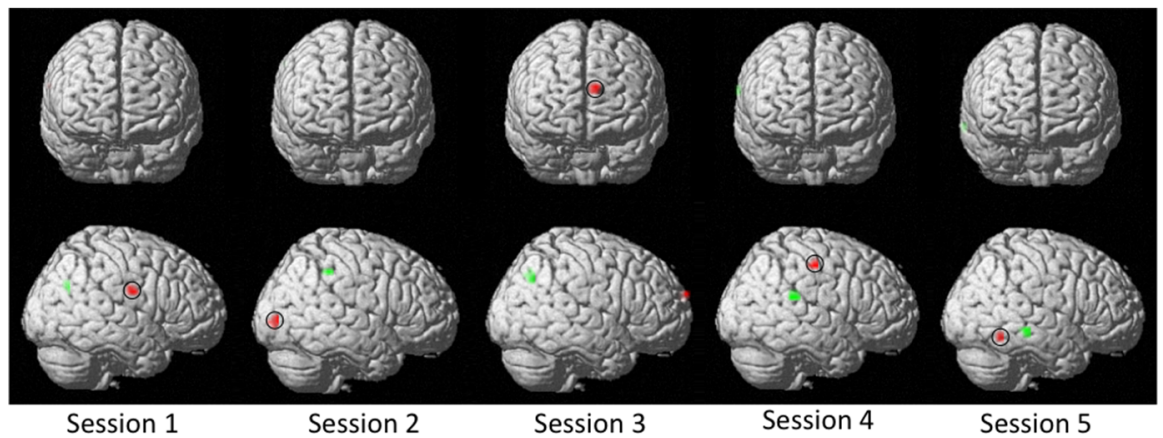

Fig. 4 The fNIRS channel locations of $\mathrm{ROI}^{F}$ and $\mathrm{ROI} \mathrm{I}^{R}$ for the participant with ASD who received real-FB at five training sessions are illustrated. The red dots (with black circles) represent the $\mathrm{ROI}^{F}$ channel and the green dots represent the $\mathrm{ROI}^{R}$ channel. As shown in the figure, the positions of the $\mathrm{ROI}^{F}$ are not necessarily consistent across sessions.

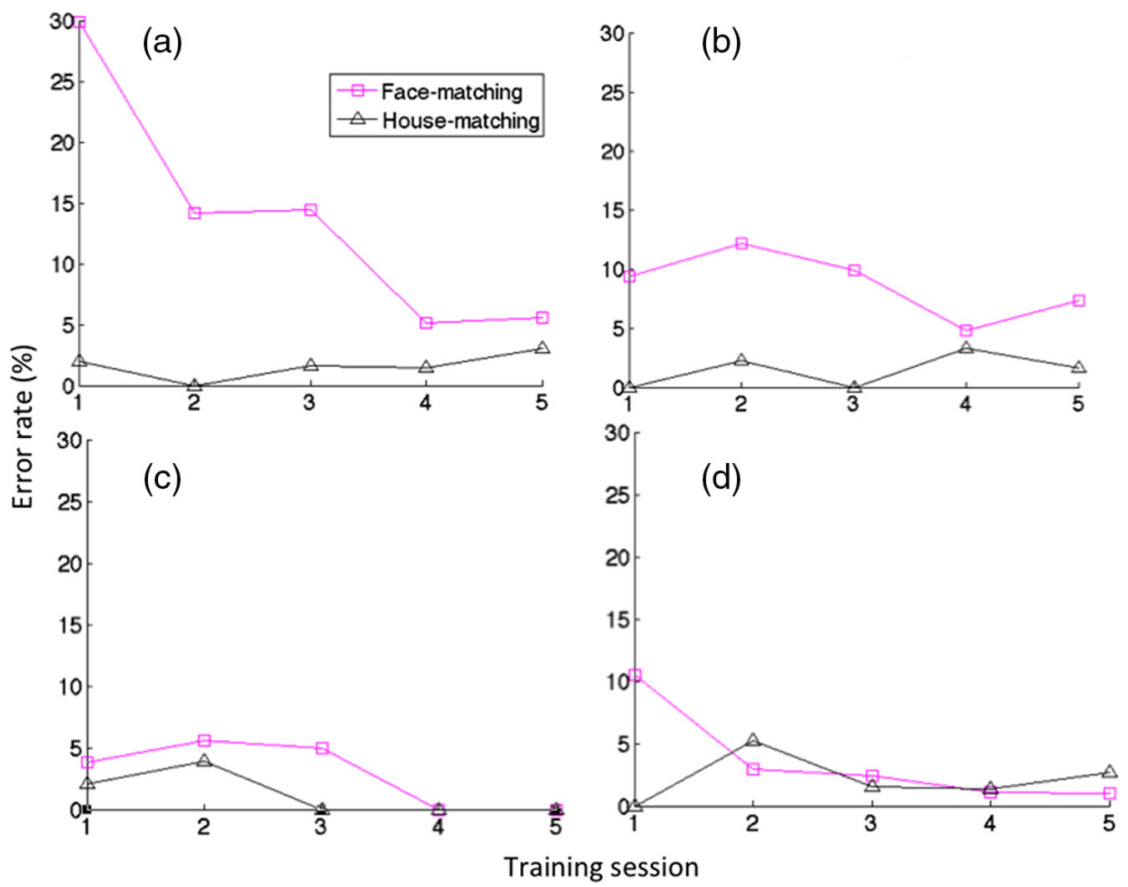

Fig. 5 Percentage error rate over the course of five training sessions for (a) the participant with ASD who received real-FB, (b) the participant with ASD who received sham-FB, (c) the TD participant who received real-FB, and (d) the TD participant who received sham-FB.

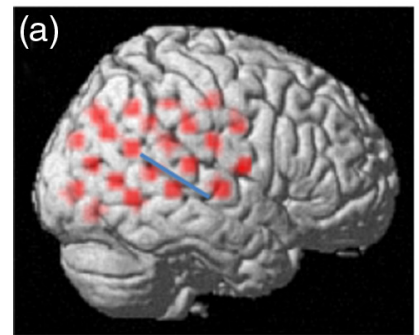

(b)
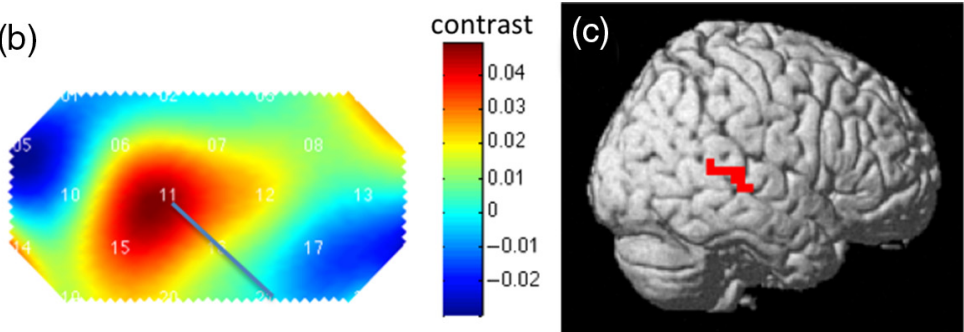

Fig. $6 \mathrm{ROI}$ analysis procedure. (a) An fNIRS channel location of a representative participant for a scan session and (b) its corresponding contrast map. (c) The voxel location of the ROI selected for offline analysis. 


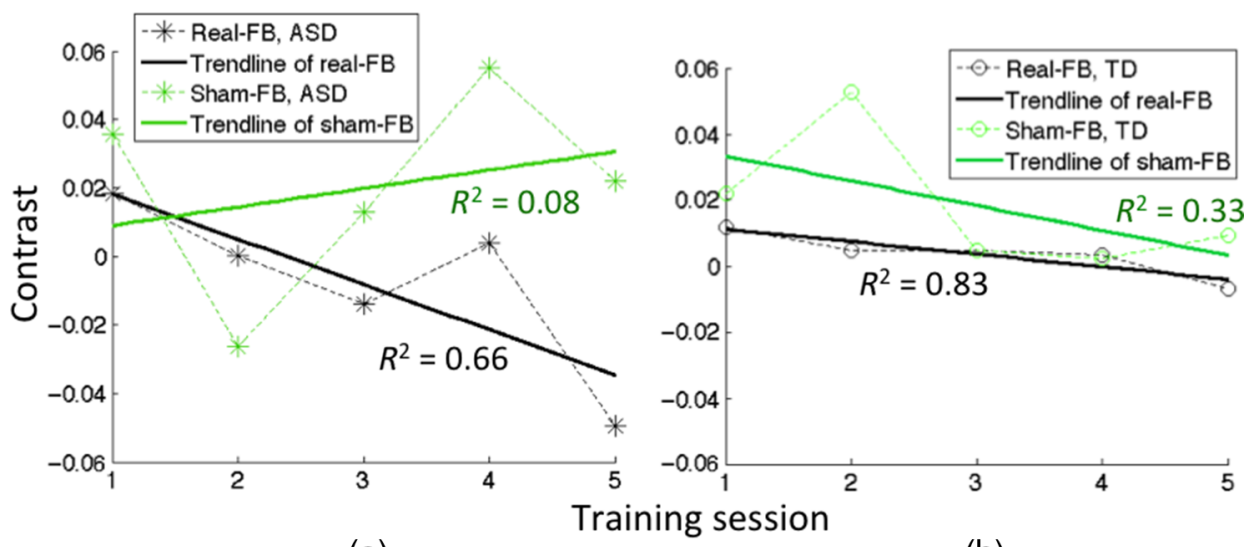

(a)

(b)

Fig. 7 Contrast values between MF and the static HP conditions at the ROI across five training sessions for (a) ASD participants and (b) TD participants.

the trend line for the participant with ASD receiving real-FB is also negative, which is similar to the slopes of the TD participants, and the absolute value of the slope was larger than both TDs', which indicates a trend of normalization of the brain activity. The slope of the trend line for the participant with ASD receiving sham-FB, however, is positive, and the contrast values of the five training sessions are scattered.

\section{Discussion}

In this study, we report, for the first time, the feasibility of using fNIRS-based neurofeedback training for children with ASD. Specifically, we developed a method to study the physiological self-regulation of [HbO] using real-time feedback of the fNIRS signal. Participants were engaged in a facial-identity recognition training program in which implicit reinforcement was given based on the participant's brain activity and behavioral performance. The paradigm is illustrated with initial data from four participants, two of whom had a confirmed diagnosis of ASD and two of whom were TD adolescents. One participant with ASD and one TD participant received real-FB during the training, and the other two received sham-FB. All four participants showed improved facial recognition performance (Fig. 3). In addition, the participants with real-FB showed more improvement compared with the participants who received sham-FB, particularly in participants with ASD. These preliminary results suggest that fNIRS-based real-FB could be used to enhance therapeutic intervention in children with ASD.

The fNIRS channel locations corresponding to the $\mathrm{ROI}^{F}$ and $\mathrm{ROI}^{R}$ for the participants with ASD were scattered, as shown in Fig. 4. Studies indicate that individuals with ASD "see" faces utilizing different neural systems as compared with TD individuals. In fact, several different patterns of individual-specific, scattered activations have been reported in individuals with ASD across studies. ${ }^{36,45}$ The results reported here are consistent with those findings.

A learning effect was observed in all four participants over the course of five training sessions, especially for the FM epochs, as shown in Fig. 5(a). The participant with ASD who received real-FB had the largest decrease in error rate among the four participants. This indicates that the real-FB may be helpful in improving behavioral performance. Interestingly, the error rates of the HM epochs were low for all participants and did not show any obvious difference between the ASD and the TD participants. The results also did not show any obvious decrease of error rate over the five training sessions, except for the TD participant who received real-FB. The participant's error rate reached zero at the third training session and was maintained for the remainder of the sessions. These results suggest that the object/house recognition abilities of the two participants with ASD were not impaired.

Compared with the participant with ASD who received sham-FB, the participant with ASD who received real-FB showed reduced activity in the right pSTS region across the five training sessions. The same trend of reduction was observed in both TD participants. As previous studies have shown, higher signal in the prefrontal and temporal cortices is considered a likely state or trait biomarker for impaired facial processing abilities in individuals with ASD. ${ }^{36,37}$ Thus, as the measured signal decreases during training, it would indicate normalization of brain activity. These results suggest that the fNIRS-based neurofeedback might help with the normalization of brain activity in children with ASD.

There are two primary strategies for self-regulation associated with neurofeedback. Explicit strategies inform the subject of a specific means for self-regulation, whereas implicit strategies provide no such instruction and allow the subject to explore different strategies or develop them without conscious perception. Although many neurofeedback studies applied explicit strategies during training, this approach has several shortcomings, especially for persons with brain disorders. For instance, it could be difficult for children or individuals with brain dysfunction to understand the provided strategy. It could also be difficult to quantitatively confirm participant performance of the suggested cognitive strategy. On the other hand, neurofeedback utilizing an implicit strategy has been reported to shape cortical network spontaneous connectivity ${ }^{46}$ and might be more effective in some circumstances. ${ }^{47}$

During methods development, particular emphasis was placed on the issue of implicit versus explicit reinforcement. The implicit strategy has several advantages for cognitive studies involving children with ASD. First, unlike emotional or motor tasks, for which it is comparatively easy to develop strategies for self-regulation, it is relatively difficult to give specific selfregulation strategies to participants during a cognitive task, such as the face identity recognition task used here. Second, some individuals with ASD may have difficulty in understanding 
suggested or required cognitive strategies. Third, the training task used in the study was an interactive computer game adopted from the LFI intervention program, which itself is challenging for children with ASD who have facial recognition deficits. Implicit reinforcement can avoid overwhelming the participant's cognitive load relative to explicit reinforcement requiring divided attention because of these challenges. We thus applied implicit reinforcement in this study — we did not introduce any specific strategies to guide the participants to gain the bonus score.

The sample size described here is inadequate for performing statistical analyses and thus for assessing the ability of fNIRS neurofeedback to address ASD symptoms. Although the results from our four participants were encouraging, more data are clearly needed to demonstrate the efficacy of the method. We have since recruited over 20 participants with ASD for a followup study to explore the efficiency of this method. The data collection is completed and the analysis is ongoing.

In summary, the method presented here is a potential tool for enhancing therapeutic intervention in children with ASD. The results suggest that providing fNIRS-based neurofeedback might enhance facial recognition performance after a short period of cognitive training. While our current study focused on improving facial recognition for children with ASD, a similar design could potentially be used to improve other cognitive functions or be used for other affected populations.

\section{Acknowledgments}

This study was supported by Autism Speaks (Grant No. \#8681).

\section{References}

1. R. Coben, M. Linden, and T. E. Myers, "Neurofeedback for autistic spectrum disorder: a review of the literature," Appl. Psychophysiol. Biofeedback 35(1), 83-105 (2010).

2. R. Christopher deCharms et al., "Control over brain activation and pain learned by using real-time functional MRI," Proc. Natl. Acad. Sci. U.S.A., 102(51), 18626-18631 (2005).

3. J. Cowan and L. Markham. "EEG biofeedback for the attention problems of autism: a case study," in Annual Meeting of the Association for applied Psychophysiology and Biofeedback (1994).

4. M. E. J. Kouijzer et al., "Neurofeedback improves executive functioning in children with autism spectrum disorders," Res. Autism Spectr. Disord. 4(1), 145-162 (2009).

5. E. V. C. Friedrich et al., "Brain-computer interface game applications for combined neurofeedback and biofeedback treatment for children on the autism spectrum," Front. Neuroeng. 7, 15-21 (2015).

6. A. Caria and S. de Falco, "Anterior insular cortex regulation in autism spectrum disorders," Front. Behav. Neurosci. 9, 38 (2015).

7. D. Oosting and B. Vander Wyk, "Functional magnetic resonance imaging," Encycl. Autism Spectr. Disord. (2015).

8. J. Kamiya, "Conscious control of brain waves," Psychol. Today 1968(1), 56-60 (1968).

9. B. Spilker et al., "Visual evoked responses in participants trained to control alpha rhythms," Psychophysiology 5(6), 683-695 (1969).

10. S. S. Yoo et al., "Brain-computer interface using fMRI: spatial navigation by thoughts," NeuroReport 15(10), 1591-1595 (2004).

11. N. Weiskopf et al., "Principles of a brain-computer interface (BCI) based on real-time functional magnetic resonance imaging (fMRI)," IEEE Trans. Biomed. Eng. 51(6), 966-970 (2004).

12. R. Christopher deCharms, "Application of real-time fMRI," Nat. Rev. Neurosci. 9(9), 720-729 (2008).

13. M. Mihara et al., "Neurofeedback using real-time near-infrared spectroscopy enhances motor imagery related cortical activation," PloS One 7(3), e32234 (2012).
14. S.E. Kober et al., "Voluntary modulation of hemodynamic responses in swallowing related motor areas: a near-infrared spectroscopy-based neurofeedback study," PLoS One 10(11), e0143314 (2015).

15. N. Liu et al. "NIRS-based hyperscanning reveals inter-brain neural synchronization during cooperative Jenga game with face-to-face communication," Front. Hum. Neurosci. 10 (2016).

16. M. Mihara et al., "Near-infrared spectroscopy-mediated neurofeedback enhances efficacy of motor imagery-based training in poststroke victims: a pilot study," Stroke J. Cereb. Circ. 44(4), 1091-1098 (2013).

17. S. E. Kober et al., "Near-infrared spectroscopy based neurofeedback training increases specific motor imagery related cortical activation compared to sham feedback," Biol. Psychol. 95, 21-30 (2014).

18. S. H. Lee et al., "Neuro-feedback using real-time near infrared spectroscopy enhances brain plasticity during treadmill walking: a pilot study," Paper presented at the 2015 IEEE Int. Conf. on Advanced Intelligent Mechatronics (AIM), pp. 437-440 (2015).

19. K. Sakatani et al., "NIRS-based neurofeedback learning systems for controlling activity of the prefrontal cortex," Adv. Exp. Med. Biol. 789, 449-454 (2013).

20. A. M. Marx et al., "Near-infrared spectroscopy (NIRS) neurofeedback as a treatment for children with attention deficit hyperactivity disorder (ADHD)-a pilot study," Front. Hum. Neurosci. 134, 153-159 (2015).

21. S. M. Hosseini et al., "Task-based neurofeedback training: a novel approach toward training executive functions," NeuroImage 134, 153-159 (2016).

22. G. Strangman et al., "A quantitative comparison of simultaneous BOLD fMRI and NIRS recordings during functional brain activation," NeuroImage 17(2), 719-731 (2002).

23. V. Y. Toronov, X. Zhang, and A. G. Webb, "A spatial and temporal comparison of hemodynamic signals measured using optical and functional magnetic resonance imaging during activation in the human primary visual cortex," NeuroImage 34(3), 1136-1148 (2007).

24. X. Cui et al., "A quantitative comparison of NIRS and fMRI across multiple cognitive tasks," NeuroImage 54(4), 2808-2821 (2011).

25. G. Dawson, S. J. Webb, and J. McPartland, "Understanding the nature of face processing impairment in autism: insights from behavioral and electrophysiological studies," Dev. Neuropsychol. 27(3), 403-424 (2005).

26. R. T. Schultz, "Developmental deficits in social perception in autism: the role of the amygdala and fusiform face area," Int. J. Dev. Neurosci. 23(2), 125-141 (2005).

27. J. M. DeGutis et al., "Functional plasticity in ventral temporal cortex following cognitive rehabilitation of a congenital prosopagnosic," J. Cognit. Neurosci. 19(11), 1790-1802 (2007).

28. University of Victoria, http://web.uvic.ca/ letsface/letsfaceit/.

29. J. W. Tanaka et al., "Using computerized games to teach face recognition skills to children with autism spectrum disorder: the let's face it! program," J. Child Psychol. Psychiatry 51(8), 944-952 (2010).

30. J. M. Wolf et al., "Specific impairment of face-processing abilities in children with autism spectrum disorder using the Let's Face It! skills battery," Autism Res. 1(6), 329-340 (2008).

31. C. Lord et al., "The autism diagnostic observation schedule-generic: a standard measure of social and communication deficits associated with the spectrum of autism," J. Autism Dev. Disord. 30(3), 205-223 (2000).

32. M. Rutter, A. Bailey, and C. Lord, The Social Communication Questionnaire (SCQ), Western Psychological Services, Torrance, California (2003).

33. J. N. Constantino and R. D. Todd, "Intergenerational transmission of subthreshold autistic traits in the general population," Biol. Psychiatry 57(6), 655-660 (2005).

34. Abrosoft FantaMorph, http://www.fantamorph.com.

35. NimStim face stimulus set, "Research network on early experience and brain development," http://www.macbrain.org/resource.htm.

36. K. Pierce et al., "Face processing occurs outside the fusiformface area' in autism: evidence from functional MRI," Brain 124(10), 2059-2073 (2001).

37. G. Golarai, K. Grill-Spector, and A. L. Reiss, "Autism and the development of face processing," Clin. Neurosci. Res. 6(3), 145-160 (2006).

38. D. T. Delpy et al., "Estimation of optical pathlength through tissue from direct time of flight measurement," Phys. Med. Biol. 33(12), 1433-1442 (1988). 
Liu et al.: Optical-imaging-based neurofeedback to enhance therapeutic intervention in adolescents...

39. N. Weiskopf et al., "Principles of a brain-computer interface (BCI) based on real-time functional magnetic resonance imaging (fMRI)," IEEE Trans. Biomed. Eng. 51, 966-970 (2004).

40. A. Caria et al., "Regulation of anterior insular cortex activity using real-time fMRI," NeuroImage 35, 1238-1246 (2007).

41. G. Rota et al., "Selfregulation of regional cortical activity using realtime fMRI: the right inferior frontal gyrus and linguistic processing," Hum. Brain Mapp. 30, 1605-1614 (2009).

42. E. A. Hoffman and J. V. Haxby, "Distinct representations of eye gaze and identity in the distributed human neural system for face perception," Nat. Neurosci. 4, 80-84 (2000).

43. A. Puce et al., "Temporal cortex activation in human viewing eye and mouth movement," J. Neurosci. 18(6), 2188-2199 (1998).
44. Z. Zhen, H. Fang, and J. Liu, "The hierarchical brain network for face recognition," PLoS One 8(3), e59886 (2013).

45. A. Di Martino et al., "Functional brain correlates of social and nonsocial processes in autism spectrum disorders: an activation likelihood estimation meta-analysis," Biol. Psychiatry 65(1), 63-74 (2009).

46. M. Ramot et al., "Covert neurofeedback without awareness shapes cortical network spontaneous connectivity," Proc. Natl. Acad. Sci. U.S.A. 113(17), E2413-E2420 (2016).

47. S. E. Kober et al., "Learning to modulate one's own brain activity: the effect of spontaneous mental strategies," Front. Hum. Neurosci. 7, 20-31 (2013).

Biographies for the authors are not available. 